\title{
Research Paper: The Immediate Effect of a Textured Insole in Nonparetic Lower Limb Symmetry of Weight Bearing and Gait Parameters in Patients with Chronic Stroke
}

\author{
Mehdi Hassan Abadi ${ }^{1},{ }^{*}$ Behnam Hajiaghaee $^{1}$, Hasan Saeedi $^{1}$, Naser Amini \\ 1. Departmant of Orthotics and Prosthetics, School of Rehabilitation Sciences, Iran University of Medical Sciences, Tehran, Iran. \\ 2. Department of Neuroscience, School of Advanced Technologies in Medicine, Tehran University of Medical Sciences, Tehran, Iran.
}

citation: Hassan Abadi M, Hajiaghaee B, Saeedi H, Amini N. [The immediate effect of a textured insole in nonparetic lower limb symmetry of weight bearing and gait parameters in patients with chronic stroke (Persian)]. Journal of Rehabilitation. 2016; 17(2):64-73.

http://dx.doi.org/10.20286/jrehab-170162

dof: http://dx.doi.org/10.20286/jrehab-170162

Received: 30 Sep. 2015 Accepted: 10 Jan. 2016

\begin{abstract}
Objective Weight-bearing asymmetry is one of the main causes of balance disturbances in patients with hemiparesis and could cause standing problems and gait abnormalities for them. The purpose of this study was to investigate the immediate effects of wearing unilateral textured insoles on the symmetry of weight bearing during standing and gait parameters of patients with chronic stroke.

Materials \& Methods In this quasi-experimental study, 16 patients with hemiparesis were selected by simple non-probability sampling method. These patients had an average age(SD) of 52.12(6.94) years and their average(SD) post-injury duration was 33.12(16.4) months. Symmetry index during standing position (by using 2 equal weighting scales), step length symmetry, step length, and walking velocity (by using NeuroCom Balance Master Device) was measured in 3 conditions: without insole (barefoot), wearing textured insole with shore A-80 hardness, and textured insole with shore A-60 hardness.

Results In this study, we conducted the multivariate analysis of variance for comparing 3 test conditions and Bonferroni test for paired comparing. The symmetry of step length showed a significant difference between no insole condition and using insole with $A-80$ hardness $(P=0.004)$, as well as using $A-80$ hardness insole with $A-60$ hardness insole $(P=0.011)$. However, there was no significant difference between using no insole and using insole with $A-60$ hardness $(P=0.325)$. The results of symmetry index likened the step length results. This means that there was a significant difference between not using insole and wearing insole with $A-80$ hardness $(P=0.022)$, also between the results of wearing 2 different insoles $(P=0.019)$. However, no significant difference was observed between using no insole and using insole with $A-60$ hardness in spite of improvement in step length $(P=0.325)$. Velocity of walking and step length was not meaningfully improved in any of the conditions.

Conclusion The current study showed that obligatory use of affected limb side could improve symmetry of weight bearing in walking and standing position of patients with chronic stroke by overcoming the phenomenon of learned lack of using and correcting the failure of sending sensory signals to centers of movement controls. The results of this study showed that unilateral use of textured insole with shore A-80 in the unaffected side could immediately improve weight bearing symmetry and step length symmetry in patients with hemiparesis, but it has no effect on their walking speed and step length. Using insole with A-60 hardness did not significantly change any variables of tests. Considering the results of this study, these insoles can be used in balance exercises and walking of hemiparetic patients.
\end{abstract}

Keywords:

Stroke, Insole, Textured insole, Weight bearing, Gait

\section{* Corresponding Author:}

Behnam Hajiaghaee, PhD

Address: Department of Orthotics and Prosthetics, School of Rehabilitation Sciences, Iran University of Medical Sciences, Shahnazari St., Madar Sq. Mirdamad Blv., Tehran, Iran.

Tel: +98 (21) 22269608

E-Mail: bhajiaghaei@yahoo.com 


\title{
بررسى تأثير كفى داراى بافت برجسته بلهصورت يكطرفه بر تقارن تحمل وزن و هارامترهاى

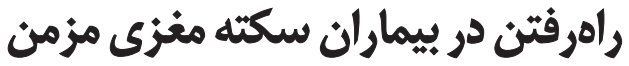

\author{
مهدى حسنآبادى'، "بهنام حاجى آقايیى'، حسن سعيدى'، ناصر امينى'
}

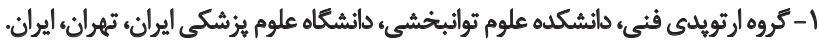

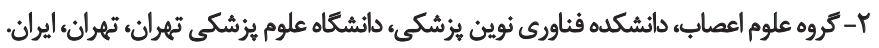

\begin{abstract}
حكبد

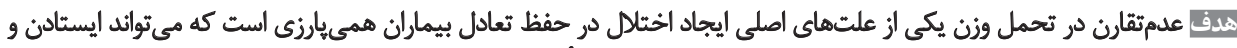

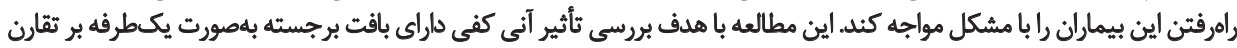

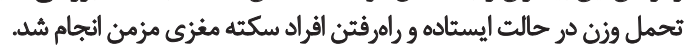

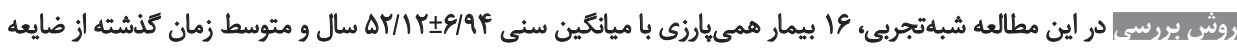
ا

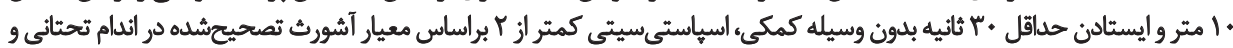

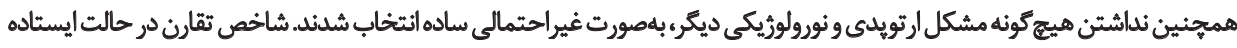

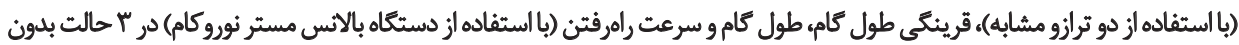

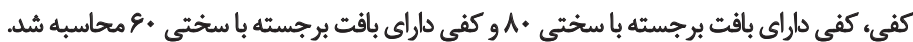

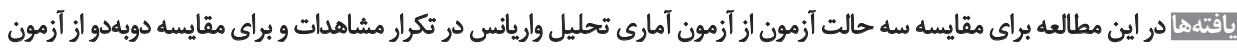

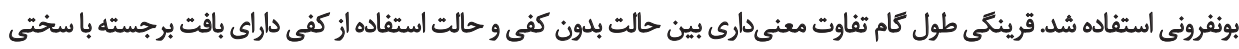

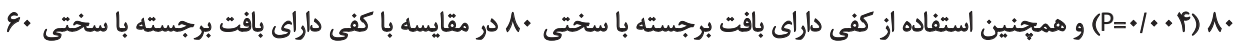

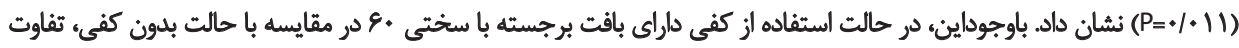

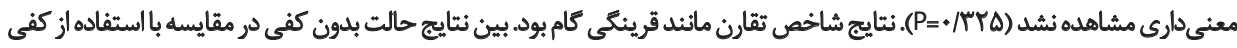

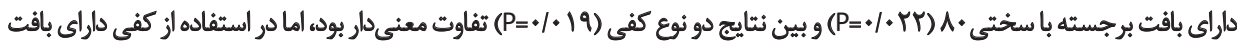

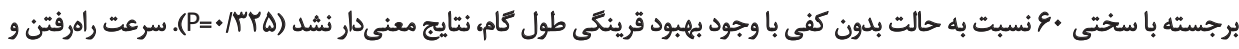

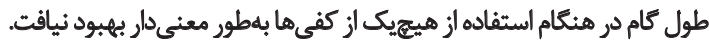

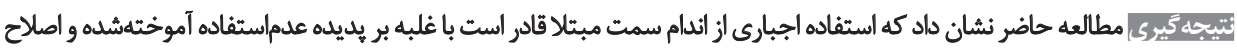

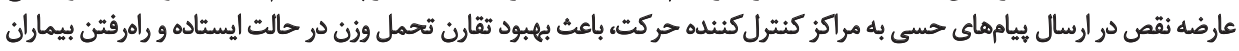

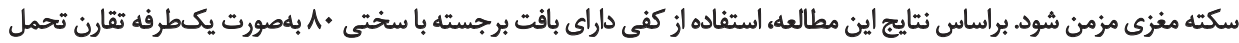

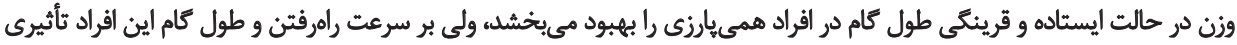

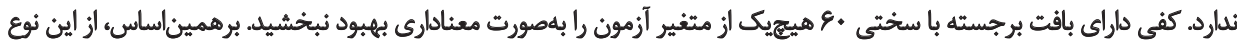

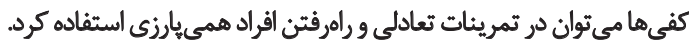

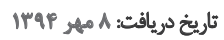

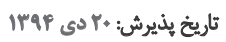

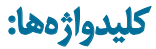

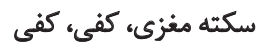

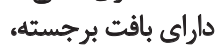
شاخص تقارن، راهرفتن 
مداخلات درمانى در زمينه ارتقاى استقالال فرد يكى از اهداف مههم

مقدمه

توانبخشى در اين بيماران است دماند].

مطالعات نشان ميدهند كه علاوهبر مطالب ذكرشده، افراد

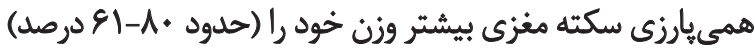

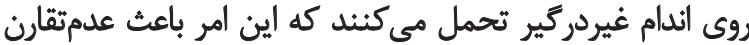

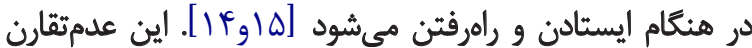

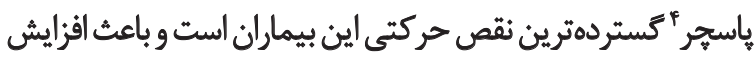

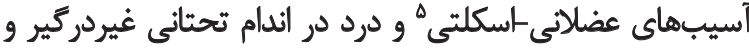

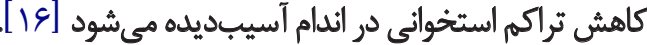

ازآنجاكه وجود تقارن در تحمل وزن بهعنوان عامل يبيشيينى كنينده

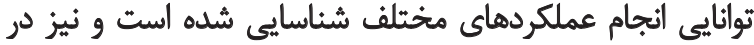

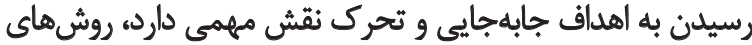

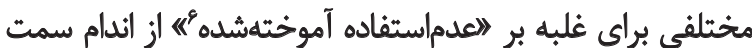

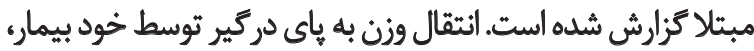

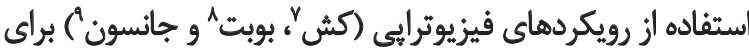

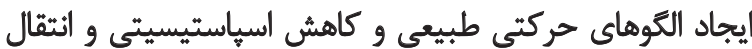

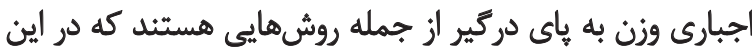

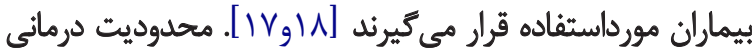

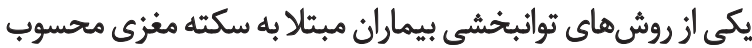

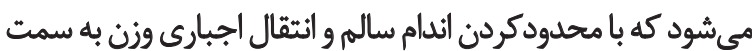
دركير (CBWS) " همراه است [11)

مطالعات مختلفى اين روش را در بهبود عملكرد اندام فوقانى

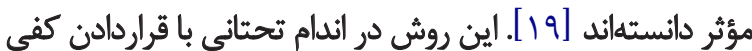

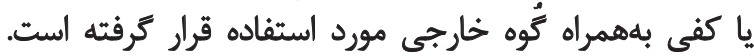

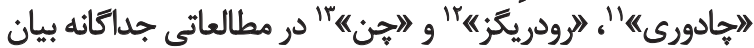

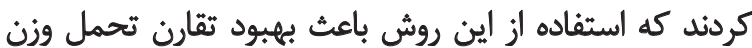

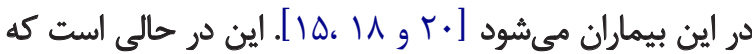

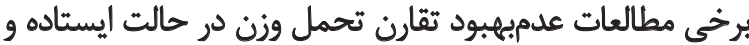

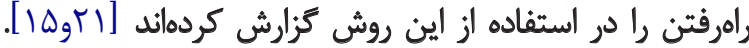

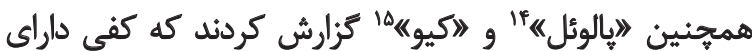

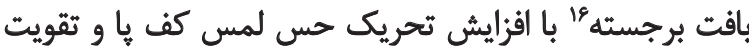

\section{Posture}

5. Musculoskeletal

6 . Learned disuse

7. Cash

8. Bobath

9. Johnson

10. Compelled Body Weight Shift (CBWS)

11. Chaudhuri

12. Rodriguez

13. Chen

14. Palluele

15. Qiu

16. Textured insole

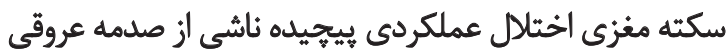

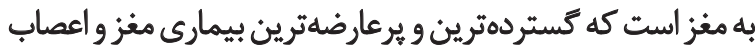

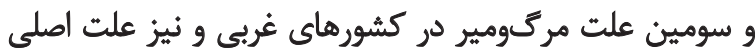

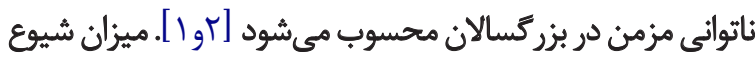

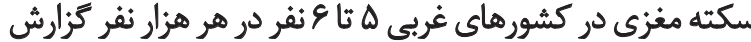
شده است [ب]

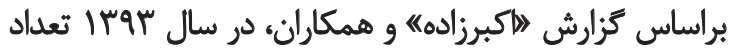

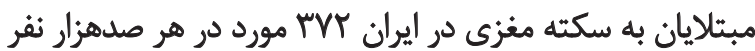

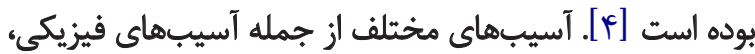

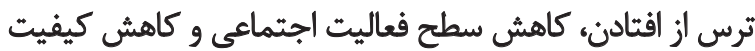

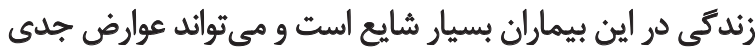

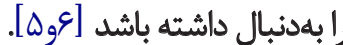

بهور كلى ميتوان كفت اختلال در كنترل تعادل و راهرفتن

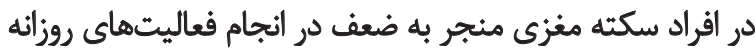

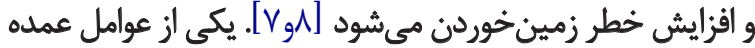

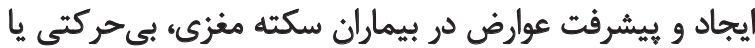

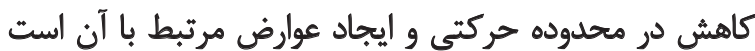

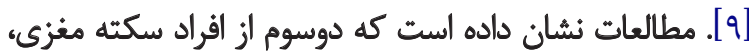

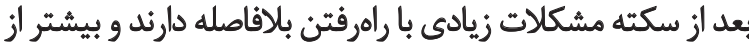

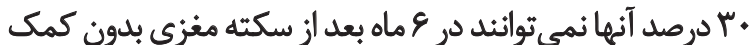

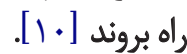

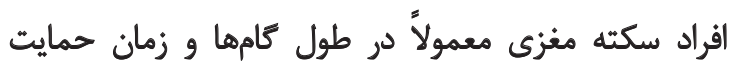

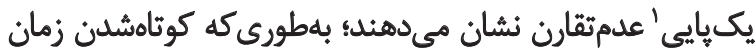

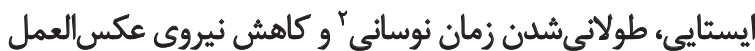

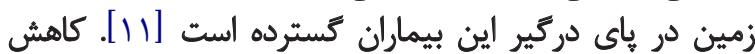

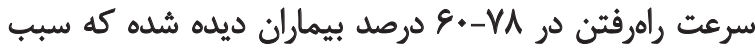

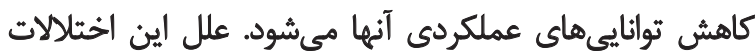

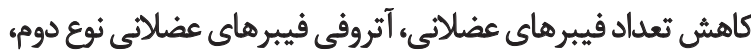

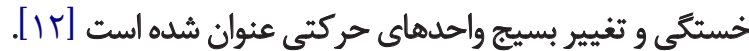

هم كاهش سرعت راهرفتن و هم افزايش خطر زمين خورين

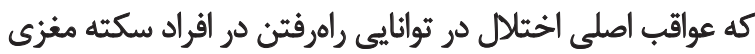

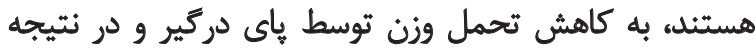

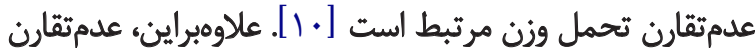

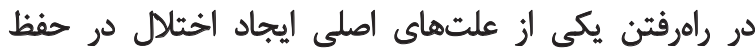

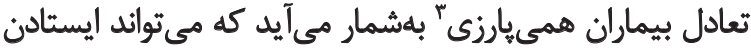

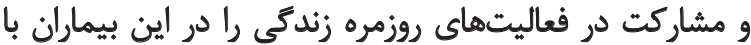

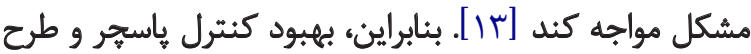

1. Single stance

2. Sewing

3. Hemiparesis 


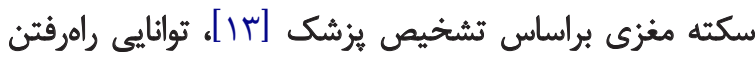

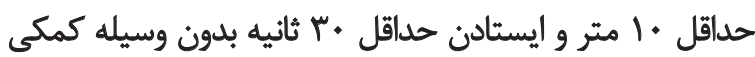

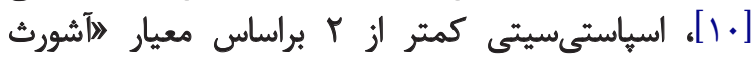

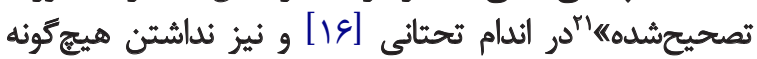

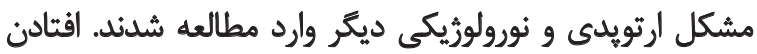

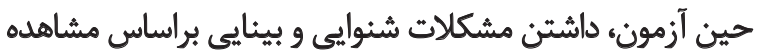

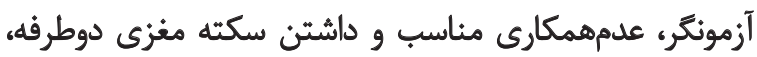
معيارهاى خروج از مطالعه بودند.

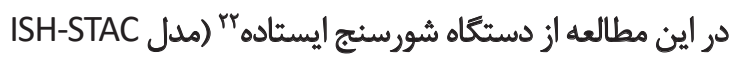

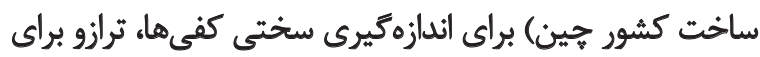

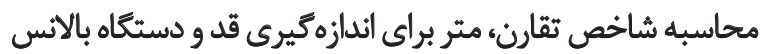

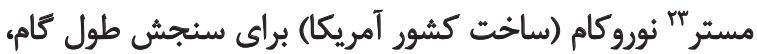

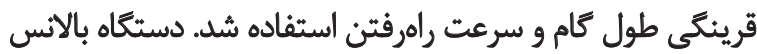

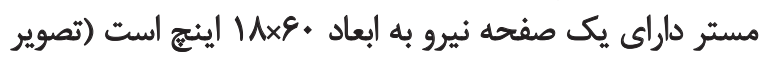

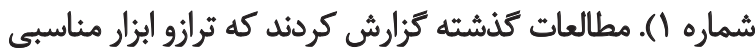

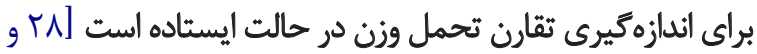

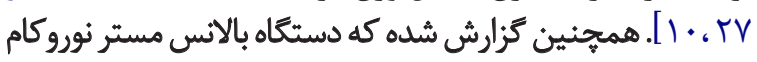

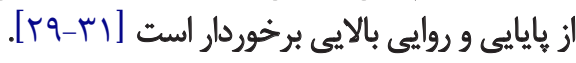

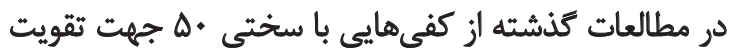

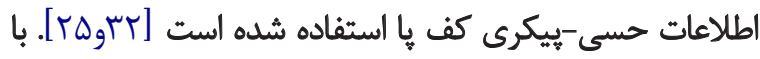

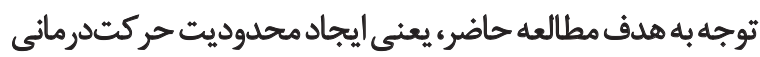

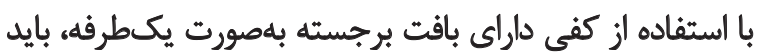

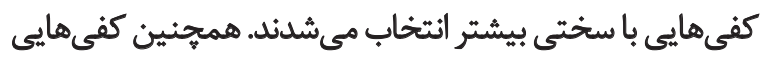

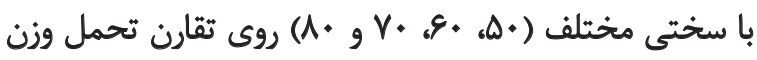

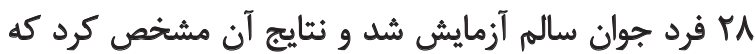

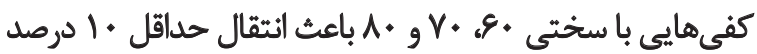

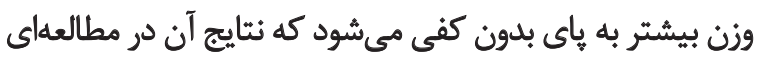
جداكانه منتشر خواهد شد.

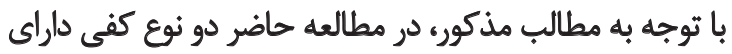

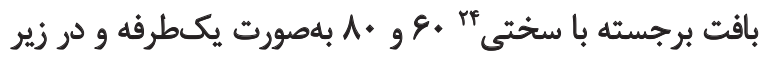

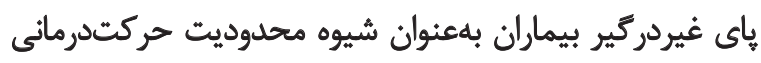

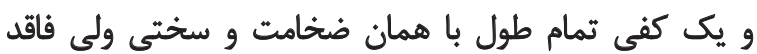

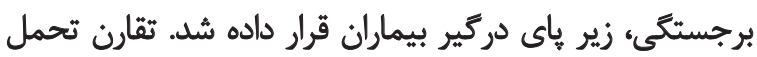

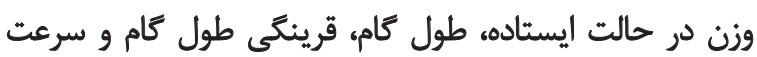
راهرفتن موردارزيابى قرار كرفت.

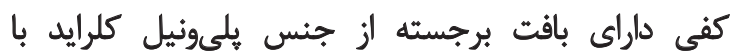

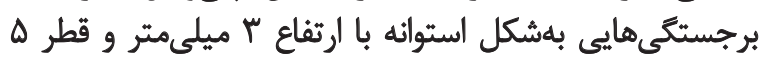

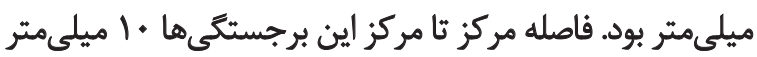

21. Modified Ashworth Scale (MAS)

22. Durometer Test Stand (DTS)

23. Balance master

24. Shore

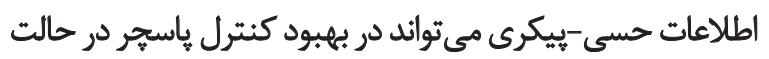

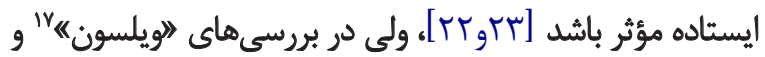

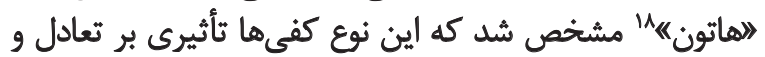

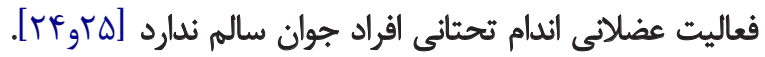

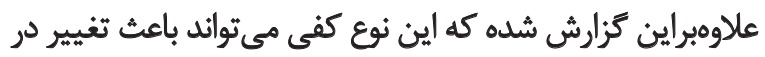
الكوى راهرفتن شود [عبارئ.

مطالعات كذشته كه كفى داراى بافت برجسته راموردبر رسيى قرار

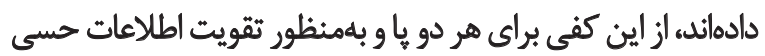

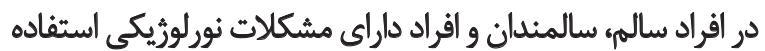

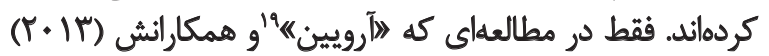

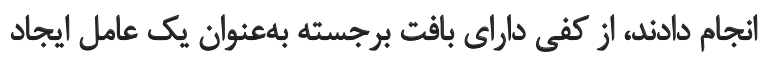

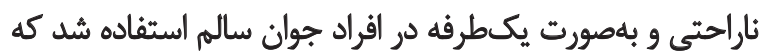

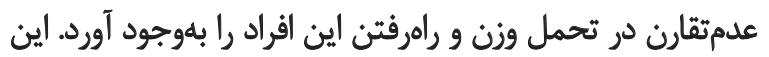

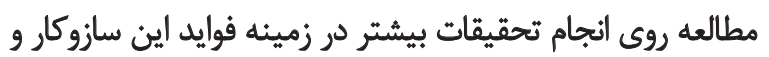

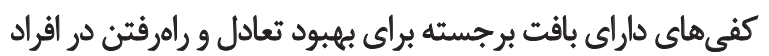
سكته مغزى همى يهارزى تأكيد مي كنيند [11)]

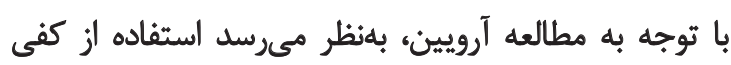

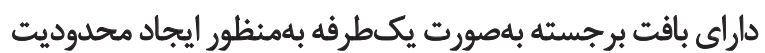

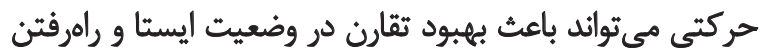

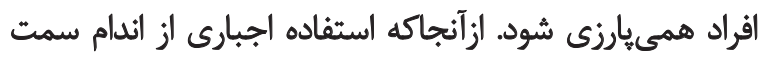

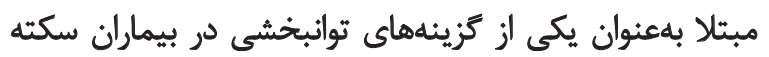

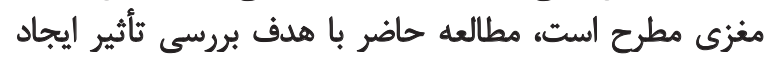

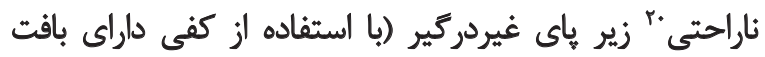

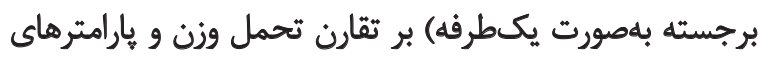

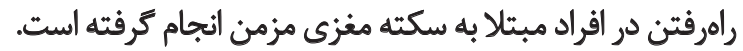

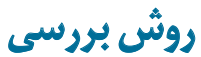

از ماز ميان بيماران مراجعكنينده به كلينيكهاي توانبخشى

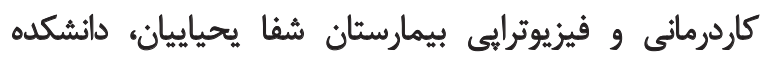

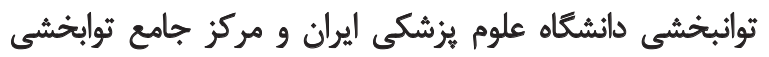
هلالاحمر، 19 بيمار سكثه مغزى شامئل

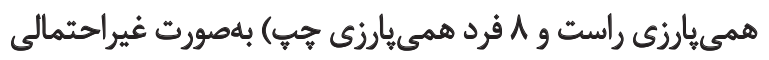

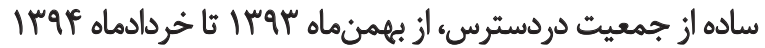

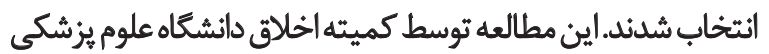

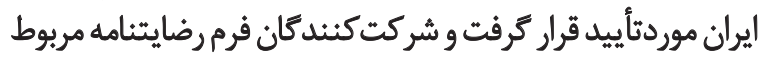

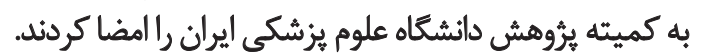

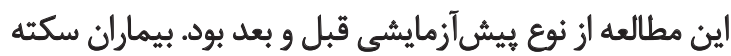

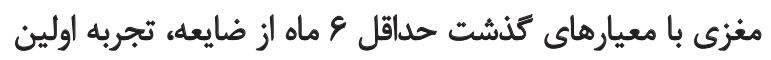

17. Wilson

18. Hatton

19. Aruin

20. Discomfort-induced approach 


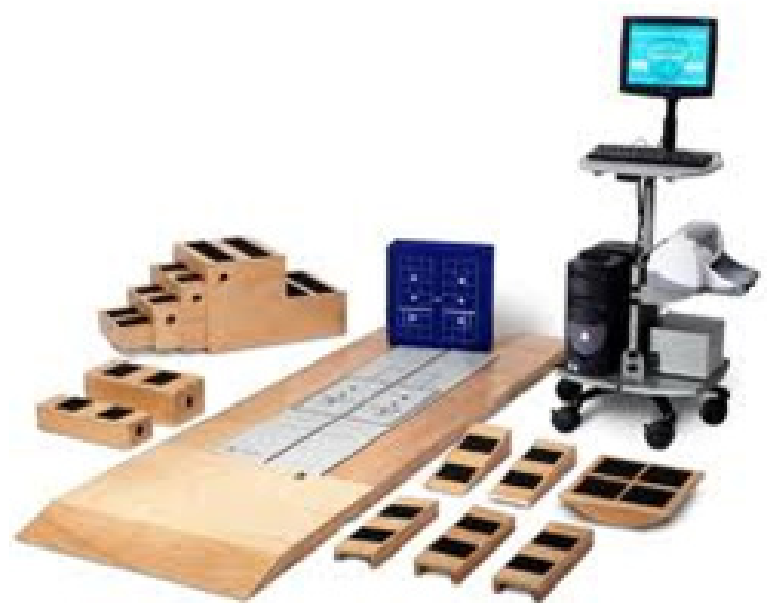

توانبخننى

تصوير 1. دستكاه بالانس مستر.

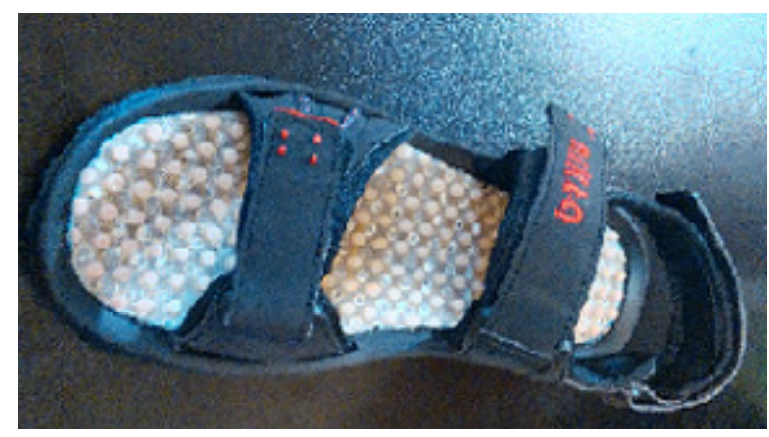

توانبخننى

تصوير r. صندل با كفى داراى بافت برجسته.

هريك از كفىها زير ياى غيردرتير و يك كفى با همان ضخامت

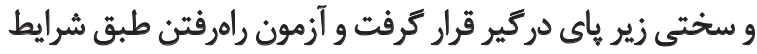

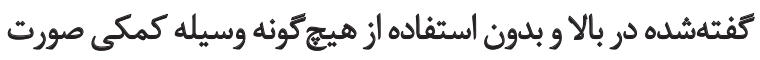

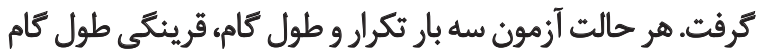

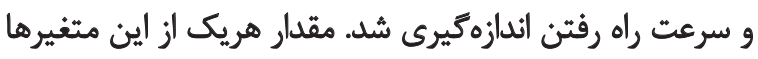

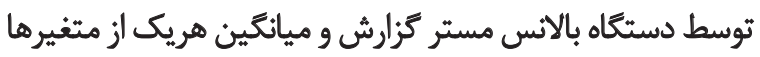
بعد از سه بار تكرار آزمون محاسبه شد.

يافتهها

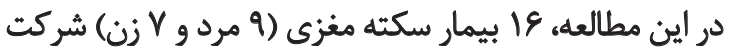

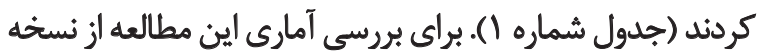

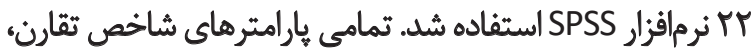

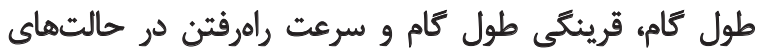

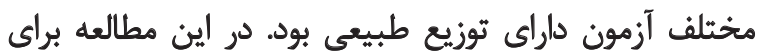

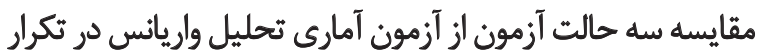

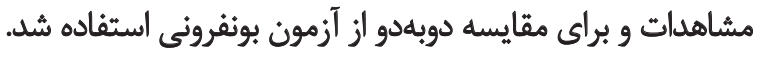

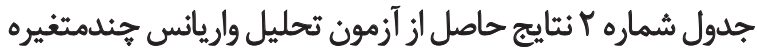

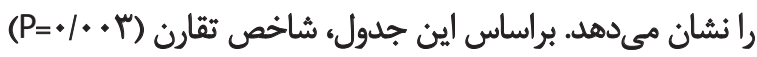

و ضخامت كفى درحدود يك ميلىمتر در نظر كرفته شد (تصوير

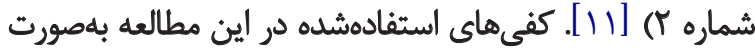

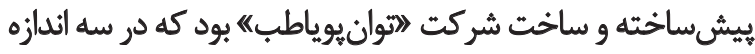

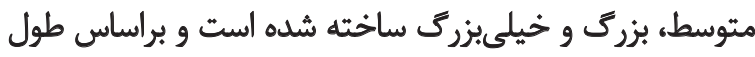

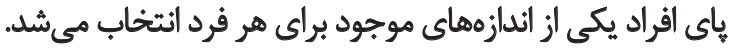

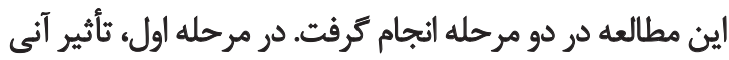

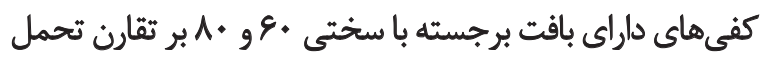

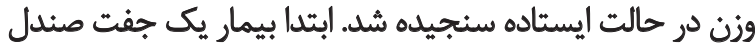

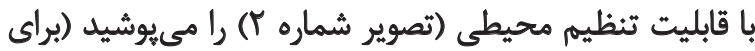

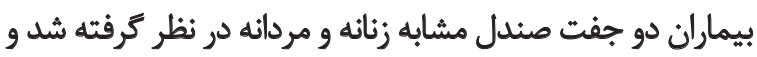

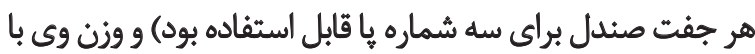

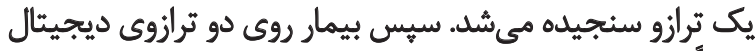

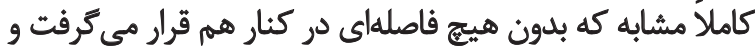

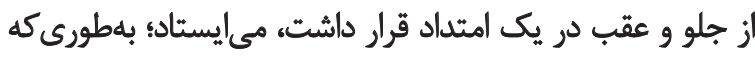

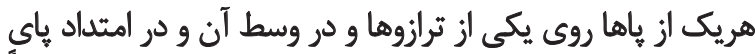

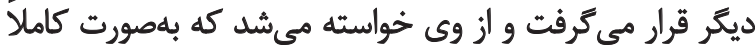

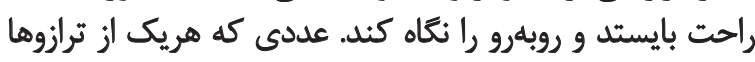

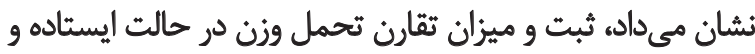

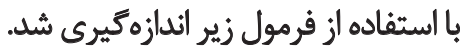
بر اساس اين فرمول، هر جه عدد بهادستآمده به صفر نزديكتر

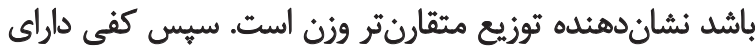

$$
\text { نيروى هاي دركير - نيروى باي غيردركير }
$$

= تقارن شاخص = $x T \times 1 \ldots$

نيروى ياى دركير+نيروى ياى غيردركير

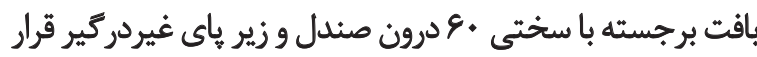

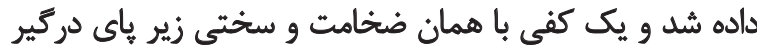

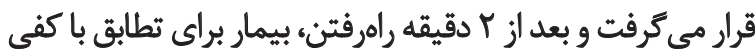

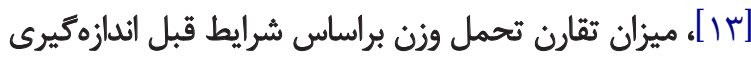

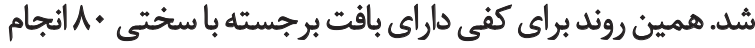

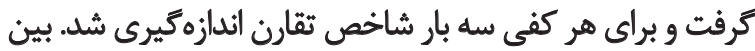

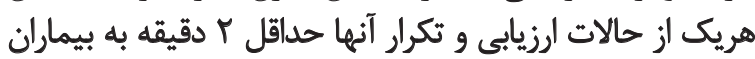

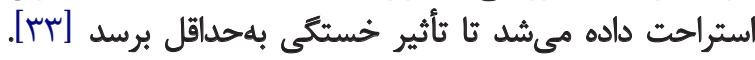

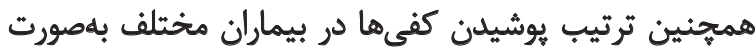
تصادفى انتخاب شد.

در مرحله دوم (آزمون طول كام، قرينكّى طول كام و سرعت

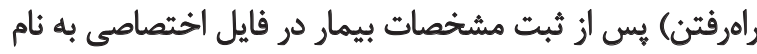

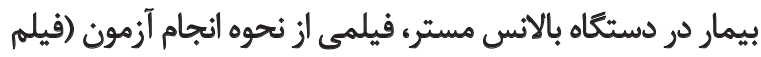

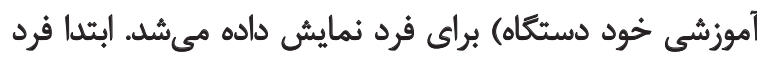

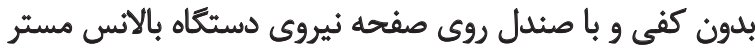

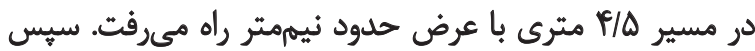


با سختى •و نسبت به حالت بدون كفى با وجود بهبود قرينكى

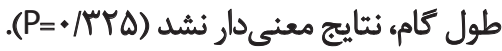

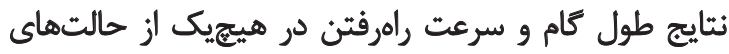

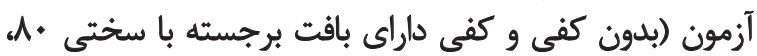

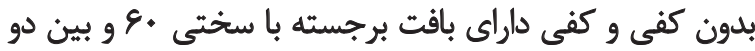

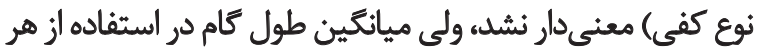

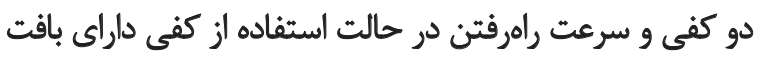

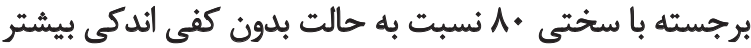
بود (جدول شماره ب).

بحث

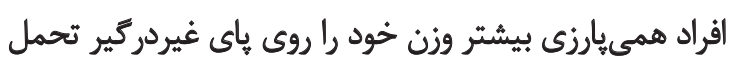

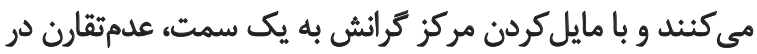

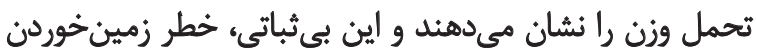

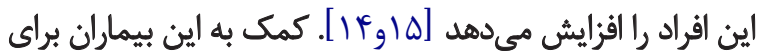

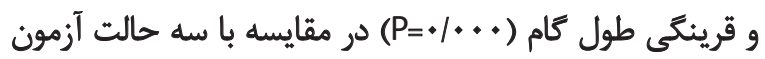
داراى تفاوت معنى فارى است.

نتايج آزمون بونفرونى براى قرينكى طول كام و شاخص تقارن

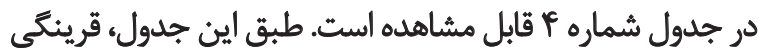

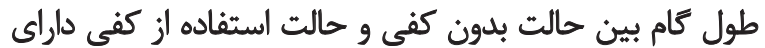

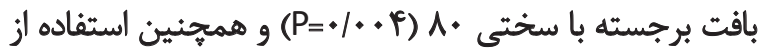

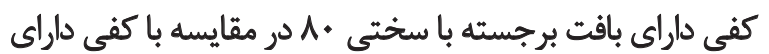

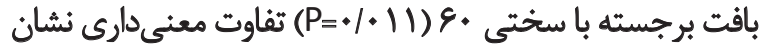

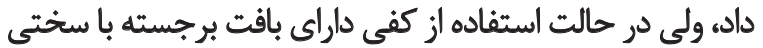

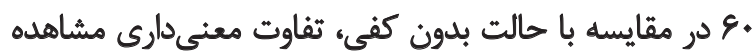

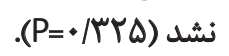

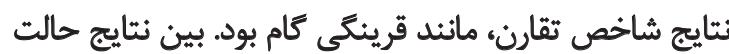

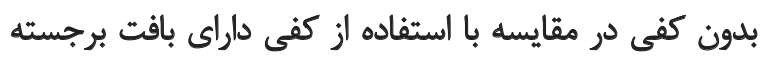

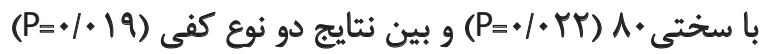

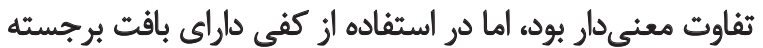

جدول أ آمار توصيفي متغيرهاي زمينهاى.

\begin{tabular}{|c|c|c|c|c|}
\hline بيشينه & كمينه & اتحراف معيار & مياتكين & مثغير \\
\hline e. & тq & e/af & $\Delta T / I T$ & سن (سال) \\
\hline ins & 181 & V/RA & $|E N|$ & قد (سانتىهتر) \\
\hline $1.8 / 5$ & $\Delta . / p$ & $18 / 09$ & $V E / \Delta V$ & جرم(كيلوكرم) \\
\hline re/ma & IVT. & $\Delta / 1 \%$ & Te/Q & شاخص جئه (كيلوكرم/مترمربع) \\
\hline$\Delta \Delta$ & 8 & IF/P. & 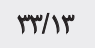 & مهت زمان سيرى ثشده (ماه) \\
\hline
\end{tabular}

توانبخنتى

جدول ب. نثايج حاصل از آزمون تحليل واريانس جندمتئيره جهت مثغيرهاي وابسته.

\begin{tabular}{|c|c|c|c|c|c|}
\hline سطح معنادارى & نسبت F & ميانكين مريعات & درجه آزادى & مجموع مريعات & منابع تغييرات (درون كروهى) \\
\hline / $1 \Delta A r$ &.$/ \Delta \Delta$ & T/ & r & $19 / 99$ & سرعت راهرقتن \\
\hline 10. & $11 / \pi \varphi$ & $9 \Delta / 1 / 9$ & r & $19.8 / M$ & قرينكى طول كام \\
\hline$\%$ & $8 / 19$ & MENA & $r$ & $E N / \Delta S$ & شاخص تقان \\
\hline.$/ r q q$ & $1 / F \Delta$ & rV/r & $r$ & AT/TE & طول كام \\
\hline
\end{tabular}

توانبخننى

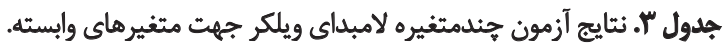

\begin{tabular}{|c|c|c|c|c|}
\hline Sig. & Hyphotesis df & $\mathbf{F}$ & value & منابع تفييرات \\
\hline.$|A A|$ & r &.$\angle A F \Delta$ & . /QYA & سرعت رامرفتن \\
\hline 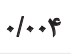 & $r$ & NFqY & . $/$ far & قرينكى طول كام \\
\hline$\cdot / 4 \cdot r$ & r &.$/ 9 V \Delta$ & - /AVA & طول كام \\
\hline .10 .9 & $r$ & 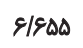 & (14) & شاخص ثقارن \\
\hline
\end{tabular}

توانبخننى 
جدول أ. آمار تحليلى شاخص تقارن و قرينكي طول كام در حالت بدون كفى، كفى داراي بافت برجسته با سختى •AA كفى داراى بافت برجسته با سختى .و.

\begin{tabular}{|c|c|c|c|c|c|}
\hline سطح معنى دارى & انحراف اسثاندارد & Mean difference & معيار & ميانكين & متغير \\
\hline$=H H^{*}$ & $r / 909$ & VIVo & $\begin{array}{l}T / .9 \pm 1 N T V \\
11 / A q \pm 9 / \%\end{array}$ & 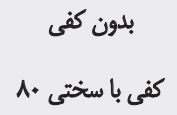 & شاخص تقان \\
\hline.$/ M T A$ & TNOA & Figay & 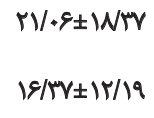 & 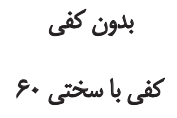 & شاخص تقان \\
\hline $.1 .19^{\circ}$ & $1 / 411$ & NYAI & $\begin{array}{l}18 / \pi V \pm 1 \% / 19 \\
11 / 19 \pm 9 / \% \Delta\end{array}$ & 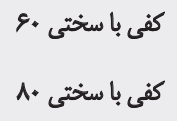 & شأصص تقارن \\
\hline .1 .0 .0 & r/AV. & ID/ATA & 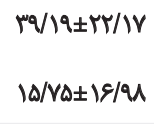 & 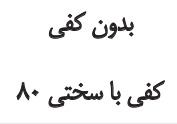 & قرينكى طول كام \\
\hline.$/ 119$ & T/TVE & entro & $\begin{array}{l}r q / 1 q \pm r r / M Y \\
r / A 1 \pm r \cdot / \Delta q\end{array}$ & كفى با بستخى +ع كثى & قرينكى طول كام \\
\hline $.1 \cdot 11 *$ & $T / T M$ & $N \cdot 94$ & $\begin{array}{l}r / A \mid \pm Y+/ \Delta q \\
|\Delta / V \Delta \pm| \& / Q \Lambda\end{array}$ & 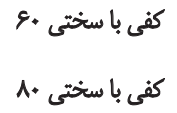 & قرينكى طول كام \\
\hline
\end{tabular}

كه اين نوع كفى ها تأثيرى بر تعادل و فعاليث عضلانى اندام تحتانى

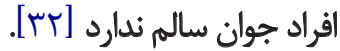

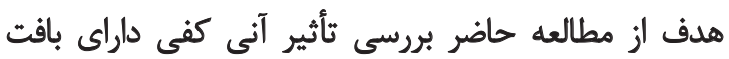

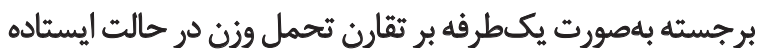

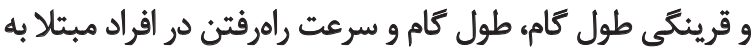
سكته مغزى مزمن بود.

استفاده از كفى داراى بافت برجسته در اين مطالعه بلهورت

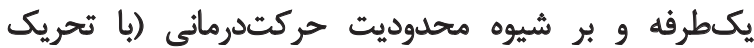

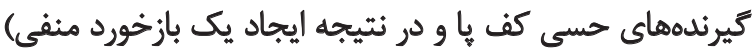

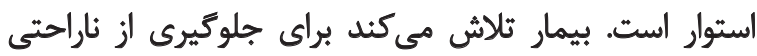

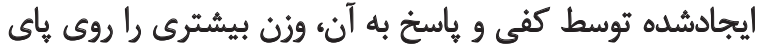

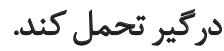

نتايج اين مطالعه نشان داد كه استفاده از كفي داراى بافت

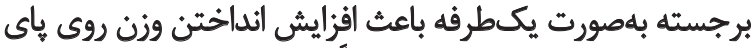

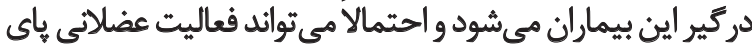

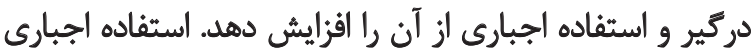

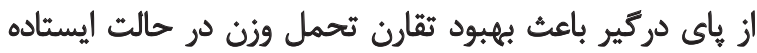

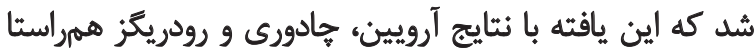

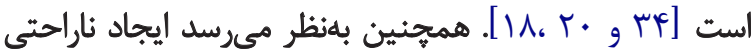

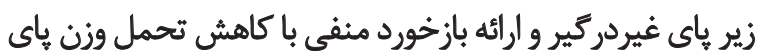

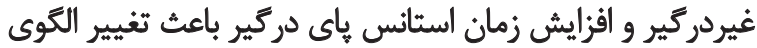

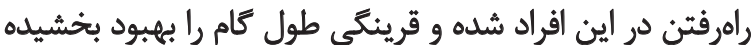

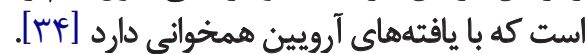

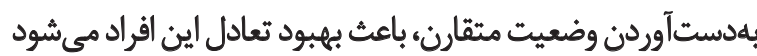

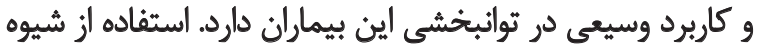

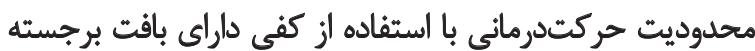

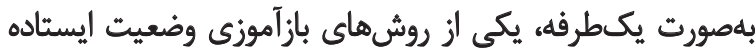
متقارن است كه در مطالعه حاضر بدان برداخته شده است بازيت

نتايج اين مطالعه نشان داد كه شاخص تقارن و قرينكى طول

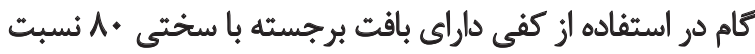

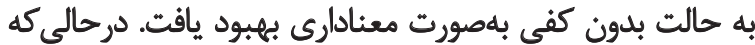

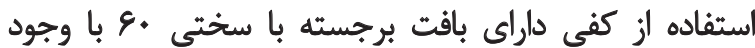

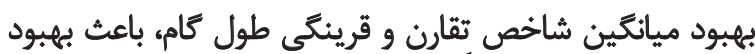

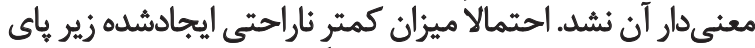

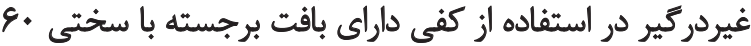

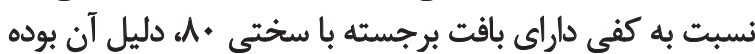

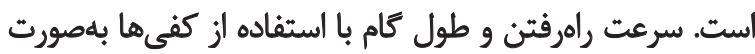

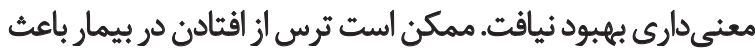
عدمبهببود سرعت راهرفتن شده است. معند است ترس

در مطالعات كذشتهنه از كفى هاى داراى بافت برجسته براى تقويت

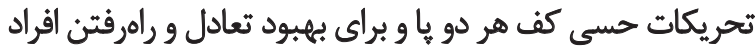

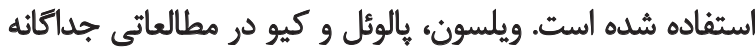

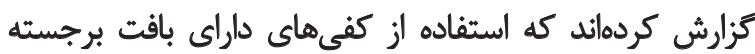

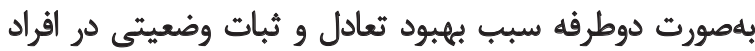

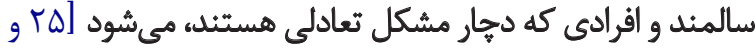

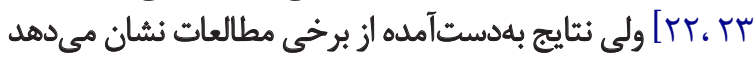




\section{References}

[1] Iranmanesh F, Gadari F. [Vitamin D and Ischemic Stroke (Persian)]. Bimonthly Journal of Hormozgan University of Medical Sciences. 2011; 15(3):178-183.

[2] Mohammadiani Nejad SE, Bavarshad SR, Majdinasab N, Kashipazha D, Oghbaei M, Mashhadizadeh N, et al. [Effect of Albumin on Clinical Function after Acute Intracerebral Hemorrhage among Hospitalized Patients in Neurology Department of Ahvaz Golestan Hospital, Iran (Persian)]. Jundishapur Scientific Medical Journal. 2013; 12(4):400-408.

[3] Iranmanesh F, Salehi M, Bakhshi H, Arab R. [Silent stroke and related risk factors (Persian)]. Journal of Gorgan University of Medical Sciences. 2013; 15(1):90-4.

[4] Akbarzadeh Baghban AR, Ahmadi Gorji S, Kavousi A, Mirkhani $\mathrm{N}$, Pourhosseingholi A. [Evaluation of Improvement of Motor Performance After Occupational Therapy in Stroke Patients Using Hurdle Model for Longitudinal Data (Persian)]. Journal of Daneshvar. 2014; 22(113):45-53.

[5] Kempen GI, Yardley L, van Haastregt JC, Zijlstra GR, Beyer N, Hauer K, et al. The Short FES-I: A shortened version of the falls efficacy scale-international to assess fear of falling. Age and Ageing. 2008; 37(1):45-50.

[6] Yardley L, Beyer N, Hauer K, Kempen G, Piot-Ziegler C, Todd C. Development and initial validation ofthe Falls Efficacy Scale-International (FES-I). Age and Ageing. 2005; 34(6):614-9.

[7] Cunha B, Alouche S, Araujo I, Freitas S. Individuals with poststroke hemiparesis are able to use additional sensory information to reduce postural sway. Neuroscience Letters. 2012; 513(1):6-11.

[8] Kamphuis JF, de Kam D, Geurts AC, Weerdesteyn V. Is weightbearing asymmetry associated with postural instability after stroke? A systematic review. Stroke Research and Treatment. 2013; 2013:692137. doi: 10.1155/2013/692137.

[9] Hossieni M, Khankeh H, Alaee S, Dibaei M. [Determine the Effect of Home Care on Complication Resulting from Musculoskeletal System Immobility of Stroked Patients (Persian)]. Journal of Rehabilitation. 2004; 5(4):35-42.

[10] Sungkarat S, Fisher BE, Kovindha A. Efficacy of an insole shoe wedge and augmented pressure sensor for gait trainingin individuals with stroke: A randomized controlled trial. Clinical Rehabilitation. 2011; 25(4):360-9.

[11] Aruin AS, Kanekar N. Effect of a textured insole on balance and gait symmetry. Experimental Brain Research. 2013; 231(2):201-8.

[12] Akbari A, Karimi H, Kazem Nejad A, Ghabaii M. [The effect of strengthening exercises on biomechanical parameters of gait in chronic hemi paresis following stroke (Persian)]. Journal of Qazvin University of Medical Sciences. 2005; 9(36):8-15.

[13] Hamedi D, Lajevardi L, Ghomashchi H, Binesh M, Taghizadeh G. [Effects of constraint induced movement therapy technique using wedge on weight bearing symmetry and functional balance in chronic hemiparesis patients (Persian)]. Koomesh. 2013; 14(3):342-9.

[14] Marigold DS, Eng JJ. The relationship of asymmetric weight-bearing with postural sway and visual reliance in stroke. Gait \& Posture. 2006; 23(2):249-55.

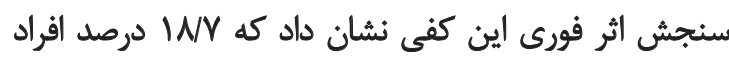

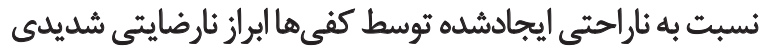

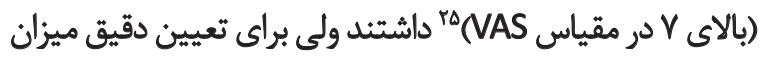

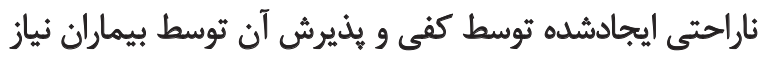

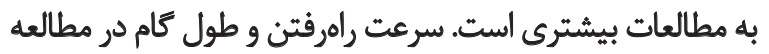

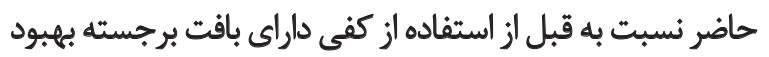

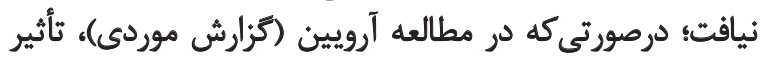

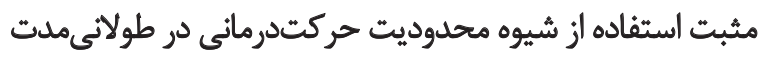

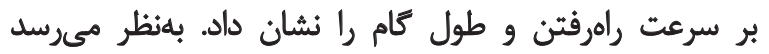

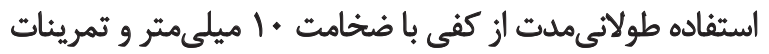

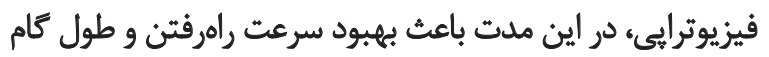

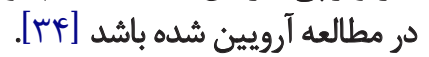

در نهايت مى توان نتيجه كرفت كه استفاده از كفى داراى بافت

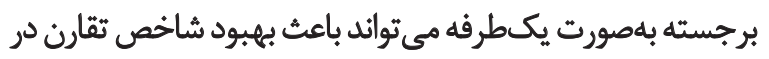

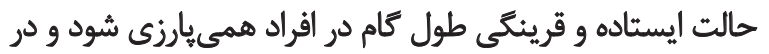

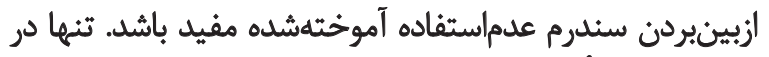

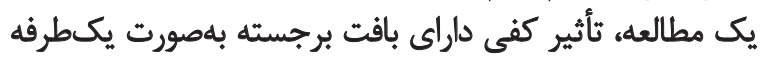

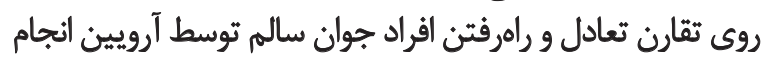
شده كه نتايج آن با يافتههاى مطالعه حاضر همسو است.

\section{نتيجه}

مطالعه حاضر نشان داد كه استفاده اجبارى از اندام سمت مبتّلا

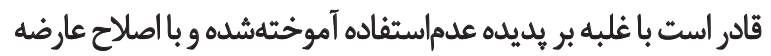

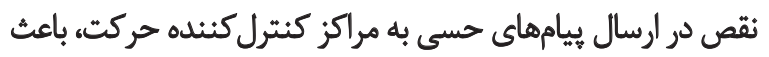

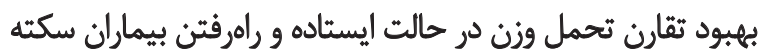

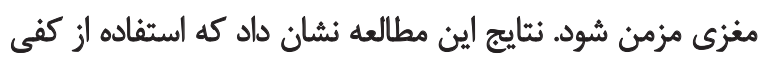

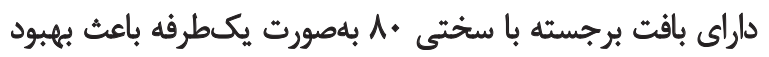

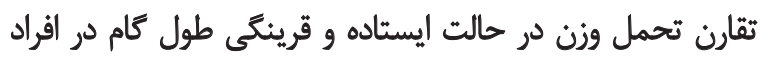

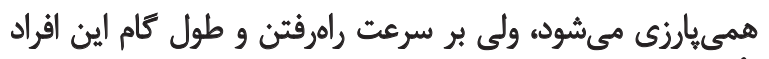

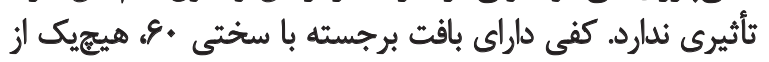

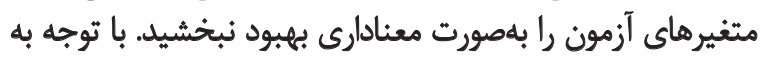

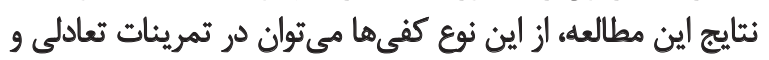
راهرفتن افراد همى يارزى استفاده كرد.

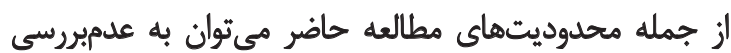

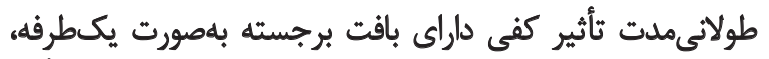

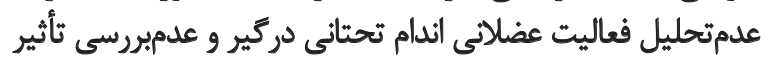

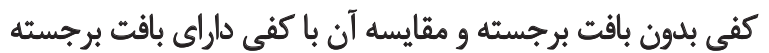

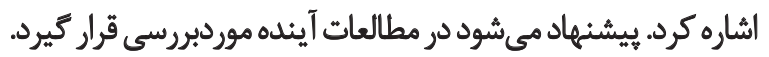

25. Visual Analog Scale (VAS) 
[15] Chen CH, Lin KH, Lu TW, Chai HM, Chen HL, Tang PF, et al. Immediate effect of lateral-wedged insole on stance and ambulation after stroke. American Journal of Physical Medicine \& Rehabilitation. 2010; 89(1):48-55.

[16] Hendrickson J, Patterson KK, Inness EL, Mcllroy WE, Mansfield A. Relationship between asymmetry of quiet standing balance control and walking post-stroke. Gait \& Posture. 2014; 39(1):177-81.

[17] Dickstein R, Nissan M, Pillar T, Scheer D. Foot-ground pressure pattern of standing Hemiplegic patients major characteristics and patterns of improvement. Physical Therapy. 1984; 64(1):19-23.

[18] Rodriguez GM, Aruin AS. The effect of shoe wedges and lifts on symmetry ofstance and weight bearing in hemiparetic individuals. Archives of Physical Medicine and Rehabilitation. 2002; 83(4):47882.

[19] Ostendorf CG, Wolf SL. Effect of forced use of the upper extremity of a Hemiplegic patient on changes in function: A single case design. Physical Therapy. 1981; 61(7):1022-8.

[20] Chaudhuri S, Aruin AS. The effect of shoe lifts on static and dynamic postural control in individuals with hemiparesis. Archives of Physical Medicine and Rehabilitation. 2000; 81(11):1498-503.

[21] Kitisomprayoonkul W, Cheawchanwattana S, Janchai S, E-Sepradit P. Effects of shoe lift on weight bearing in stroke patients. JournalMedical Assosiation of Thailand. 2005; 88(Suppl 4):S79-S84.

[22] Palluel E, Nougier V, Olivier I. Do spike insoles enhance postural stability and plantar-surface cutaneous sensitivity in the elderly? Age. 2008; 30(1):53-61.

[23] Qiu F, Cole MH, Davids K, Hennig E, Silburn P, Netscher H, et al. Enhanced somatosensory information decreases postural sway in older people. Gait \& Posture. 2012; 35(4):630-5.

[24] Hatton AL, Dixon J, Rome K, Martin D. Standing on textured surfaces: effects on standing balance in healthy older adults. Age and Ageing. 2011; 40(3):363-8.

[25] Wilson ML, Rome K, Hodgson D, Ball P. Effect of textured foot orthotics on static and dynamic postural stability in middle-aged females. Gait \& Posture. 2008; 27(1):36-42.

[26] Dixon J, Hatton A, Robinson J, Gamesby-Iyayi H, Hodgson D, Rome $\mathrm{K}$, et al. Effect of textured insoles on balance and gait in people with multiple sclerosis: An exploratorytrial. Physiotherapy. 2014; 100(2):142-9.

[27] Bohannon RW, Larkin PA. Lower extremity weight bearing under various standing conditions in independently ambulatory patients with hemiparesis. Physical Therapy. 1985; 65(9):1323-5.

[28] Pyöriä O, Era P, Talvitie U. Relationships between standing balance and symmetry measurements in patients following recent strokes ( $\leq 3$ weeks) or older strokes ( $\geq 6$ months). Physical Therapy. 2004; 84(2):128-36.

[29] Liston RA, Brouwer BJ. Reliability and validity of measures obtained from stroke patients using the Balance Master. Archives of Physical Medicine and Rehabilitation. 1996; 77(5):425-30.

[30] Newstead AH, Hinman MR, Tomberlin JA. Reliability of the Berg Balance Scale and balance master limits of stability tests for individuals with brain injury. Journal of Neurologic Physical Therapy. 2005; 29(1):18-23.
[31] Chien CW, Hu MH, Tang PF, Sheu CF, Hsieh CL. A comparison of psychometric properties of the smart balance master system and the postural assessment scale for stroke in people who have had mild stroke. Archives of Physical Medicine and Rehabilitation. 2007; 88(3):374-80.

[32] Hatton AL, Dixon J, Rome K, Newton JL, Martin DJ. Altering gait by way of stimulation of the plantar surface of the foot: the immediate effectof wearing textured insoles in older fallers. Journal of Foot and Ankle Research. 2012; 5:11.

[33] Asghar Hosseini H, Ebrahimi E, Salavati M, Shahidi GA, Sanjari M, Gholamipour A. [Effect of symmetry improvement in weight bearing on postural stability of hemiparetic patients (Persian)]. Journal of Rehabilitation. 2008; 9(2):42-46.

[34] Aruin AS, Hanke T, Chaudhuri G, Harvey R, Rao N. Compelled weightbearing in persons with hemiparesis following stroke: The effect of a lift insert and goal-directed balance exercise. Journal of Rehabilitation Research and Development. 2000; 37(1):65-72. 
